Service social

\title{
Théorie et pratiques en organisation communautaire, sous la direction de Laval Doucet et Louis Favreau, Sillery, Presses de l’Université du Québec, 1991, 468 pages.
}

\section{Jean-Pierre Deslauriers}

Volume 40, numéro 2, 1991

Formation et évolution de la pratique en travail social

URI : https://id.erudit.org/iderudit/706538ar

DOI : https://doi.org/10.7202/706538ar

Aller au sommaire du numéro

Éditeur(s)

École de service social de l'Université Laval

ISSN

1708-1734 (numérique)

Découvrir la revue

Citer ce compte rendu

Deslauriers, J.-P. (1991). Compte rendu de [Théorie et pratiques en organisation communautaire, sous la direction de Laval Doucet et Louis Favreau, Sillery, Presses de l'Université du Québec, 1991, 468 pages.] Service social, 40(2), 153-155. https://doi.org/10.7202/706538ar d'utilisation que vous pouvez consulter en ligne.

https://apropos.erudit.org/fr/usagers/politique-dutilisation/ 
En outre, une présentation plus méthodique aurait grandement contribué à rehausser le contenu de ce livre. Quant au titre, il ne correspond pas suffisamment au contenu : l'auteur ne réussit pas réellement à analyser l'insignifiance du travail et le lecteur demeure submergé par les chiffres.

Le nombre de références toutefois est considérable : 405 notices bibliographiques qui ont été puisées principalement dans des journaux (La Presse, Le Devoir, Le Soleil, Le Journal de Montréal), des revues (L'actualité, Écologie) ainsi que des documents gouvernementaux. II est à noter que si les références avaient été données en ordre alphabétique, il aurait été plus facile de repérer les auteurs des articles, mais c'est tout de même un travail de recherche imposant et le plus grand mérite de ce livre.

Bref, le praticien qui recherche une réflexion personnelle sur le travail risque fort de trouver indigeste l'amoncellement de statistiques contenues dans cet essai. Par contre, le lecteur qui cherche des statistiques brèves sur les différents aspects du travail (politique, économique et social) a de fortes chances d'être satisfait. Ce livre pourrait avoir sa place dans la bibliothèque des professeurs et des étudiants en sciences humaines qui ont à analyser les différents problèmes sociaux liés au travail. De plus, le coût du livre est abordable.

Jeanne Bélanger

Andragogue

Hull

Théorie et pratiques en organisation communautaire, sous la direction de Laval DouCET et Louis FAVREAU, Sillery, Presses de l'Université du Québec, 1991, 468 pages.

Au cours des années 60 , les textes dont les étudiants québécois en organisation communautaire disposaient étaient la plupart du temps le fait d'auteurs américains : rarissimes étaient les travaux pertinents écrits en français. Nous plaquions donc les théories et pratiques de nos voisins du sud à la réalité qui était la nôtre, mais avec les discordances faciles à prévoir : il n'y a pas homologie des pratiques entre une société plutôt individualiste et une autre " tricotée plus serré ". La société québécoise disposait pourtant d'un important bagage d'expériences d'organisation communautaire, mais nous faisions comme si nous ne pouvions encore en tirer des leçons comme les Américains le faisaient pourtant pour eux-mêmes.

Vinrent ensuite les années 70 au cours desquelles un fort vent de dogmatisme souffla sur l'organisation communautaire; plusieurs n'avaient plus que faire de ces recettes empiriquement distillées par l'expérience et se sentaient davantage attirés par la théorie pure. L'influence américaine fut remplacée par celle du léninisme, du stalinisme, du maoïsme, et autres Enver Hoxha. II s'agissait donc de suivre la ligne juste, souvent la plus droite mais pas nécessairement la plus praticable.

II a fallu attendre les années 80 pour voir paraître les premiers livres d'organisation communautaire, mais le défi n'était pas facile à relever : il fallait relire l'expérience américaine avec laquelle nous partageons la sensibilité et une expérience semblables, mais en l'appliquant à notre contexte. Voilà la tâche à laquelle se sont attaqués avec bonheur les collègues Doucet et Favreau dans ce qui constitue le premier vrai livre québécois d'introduction à l'organisation communautaire. 
Le travail de rédaction fut colossal : pour traiter le sujet, on a fait appel aux offices de dix-huit auteurs et de dix-sept collaborateurs, issus d'une variété d'établissements universitaires, de champs disciplinaires et de champ de pratique. Cette diversité laisse pressentir le travail de coordination qu'elle a exigé. II s'agit donc d'un recueil des textes, mais préparé avec grande minutie. Habituellement, les responsables d'un recueil de textes s'adressent à des auteurs et sollicitent des articles; ils reçoivent les contributions, les entourent d'une présentation et le tour est joué. Cependant, ce livre fait exception à la règle. D'abord, les textes des différents auteurs ont été évalués, arbitrés, modifiés, comme il est d'usage de le faire dans une bonne revue. Ensuite, à mi-chemin de la rédaction, les responsables du livre ont réuni tous les auteurs et collaborateurs pour discuter du contenu, critiquer les différents articles et harmoniser les uns avec les autres. Enfin, ce volume a lentement mûri comme blé au soleil : le lecteur tient dans ses mains la troisième version du manuscrit.

Ce livre a plusieurs mérites, dont celui d'offrir une méthodologie d'organisation communautaire précise. Inspirés par les travaux de Rothman dont ils se réclament, Doucet et Favreau identifient quatre étapes dans le processus de l'organisation communautaire, soit voir, juger, agir et évaluer. À partir de cette méthodologie générale, ils définissent ensuite trois modèles d'organisation communautaire. D'abord, I'action sociale, utilisée surtout dans la défense des droits sociaux, la revendication et la protestation et qui entend établir un rapport de forces favorable aux plus pauvres; le développement local qui se retrouve dans la réalisation de projets communautaires et qui se caractérise par l'addition des forces; enfin, le planning social qui vise "la résolution des problèmes sociaux des communautés locales par une intervention étatique de proximité à partir d'appareils sociaux qui, investis de pouvoirs et de ressources pour s'attaquer aux problèmes sociaux de l'heure, interviennent sur le plan local (les CLSC) ou sur le plan municipal... » (p. 15).

Cette variété de modèles d'intervention colle à l'évolution de l'organisation communautaire au Québec. Les années 60 et 70 furent une sorte d'âge d'or de l'action sociale : on s'opposait et on défendait les droits, mais sans dépasser la demande d'une plus grande intervention de l'État pour répondre aux besoins sociaux. La fin des années 70 et les années 80 furent plus inventives de ce point de vue : non seulement les groupes communautaires continuèrent-ils de protester, mais ils mirent sur pied des organisations plus efficaces que celles de l'État. Par ailleurs, la pression que les groupes communautaires exercent depuis une vingtaine d'années a modifié l'appareil étatique : la mise sur pied des CLSC en est un exemple, tout comme la complémentarité que l'État vient de découvrir entre les groupes communautaires et ceux du réseau officiel. Ces trois modèles rendent donc compte d'une multitude d'expériences, mais pour ne pas être en reste, les auteurs prennent soin de présenter des articles portant sur des approches avec lesquelles leurs modèles partagent des similitudes, telles que I'action politique locale, l'action conscientisante et l'approche communautaire.

Si la première partie est de teneur méthodologique et historique, la deuxième présente les différents champs de pratique de l'organisation communautaire, des jeunes aux femmes en passant par les personnes âgées, les groupes ethniques et l'éducation populaire. De plus, on distingue le contexte de l'organisation communautaire selon qu'elle se déploie dans le milieu urbain et rural. Ces articles illustrent à leur manière les trois modèles que les auteurs présentent dans la première partie. Enfin, quoique moins importante numériquement mais d'un grand intérêt, la troisième partie porte sur l'organisation communautaire en Afrique et en Amérique latine. Il est évident que la transposition n'est pas automatique, mais il reste que le nord peut apprendre du sud. 
Ce volume n'est pas sans faiblesses, comme l'absence d'un index thématique pourtant si utile aux lecteurs, des récits d'expérience qui ne sont pas toujours aussi étroitement reliés qu'on aimerait à la méthodologie de l'organisation communautaire privilégiée par les deux responsables. Toutefois, en dépit de ces lacunes, ce livre représente le travail le plus achevé dont disposent actuellement les étudiants et les intervenants en organisation communautaire. En plus des indications méthodologiques intégrées, il contient une richesse $d^{\prime}$ informations historiques. Pour toutes ces raisons, ce livre constituera un jalon important dans le développement de la théorie et de la pratique de l'organisation communautaire, et un point de repère précieux dans l'évolution des mouvements sociaux.

Jean-Pierre Deslauriers

Université du Québec

Hull

NOTA. - De façon à favoriser les échanges d'idées, le Comité de la revue SERVICE SOCIAL recommande la publication de deux points de vue différents concernant cet ouvrage. En effet, une première recension soumise dès la parution du volume par monsieur Gérald Doré n'a pu être publiée en même temps que celle-ci. Elle paraitra dans notre prochain numéro: Intolérance, oppressionet intervention (1991, volume 40 numéro 3 ). 